\title{
Optimization of an Accelerometer and Gyroscope-Based Fall Detection Algorithm
}

\author{
Quoc T. Huynh, ${ }^{1}$ Uyen D. Nguyen, ${ }^{2}$ Lucia B. Irazabal, ${ }^{1}$ \\ Nazanin Ghassemian, ${ }^{1}$ and Binh Q. Tran ${ }^{1}$ \\ ${ }^{1}$ Department of Biomedical Engineering, School of Engineering, The Catholic University of America, Washington, DC 20064, USA \\ ${ }^{2}$ School of Electrical Engineering, International University, Vietnam National University, Ho Chi Minh City 70000, Vietnam \\ Correspondence should be addressed to Binh Q. Tran; tran@cua.edu
}

Received 22 September 2014; Revised 6 February 2015; Accepted 5 March 2015

Academic Editor: Alain Pauly

Copyright (C) 2015 Quoc T. Huynh et al. This is an open access article distributed under the Creative Commons Attribution License, which permits unrestricted use, distribution, and reproduction in any medium, provided the original work is properly cited.

Falling is a common and significant cause of injury in elderly adults ( $>65 \mathrm{yrs}$ old), often leading to disability and death. In the USA, one in three of the elderly suffers from fall injuries annually. This study's purpose is to develop, optimize, and assess the efficacy of a falls detection algorithm based upon a wireless, wearable sensor system (WSS) comprised of a 3-axis accelerometer and gyroscope. For this study, the WSS is placed at the chest center to collect real-time motion data of various simulated daily activities (i.e., walking, running, stepping, and falling). Tests were conducted on 36 human subjects with a total of 702 different movements collected in a laboratory setting. Half of the dataset was used for development of the fall detection algorithm including investigations of critical sensor thresholds and the remaining dataset was used for assessment of algorithm sensitivity and specificity. Experimental results show that the algorithm detects falls compared to other daily movements with a sensitivity and specificity of $96.3 \%$ and $96.2 \%$, respectively. The addition of gyroscope information enhances sensitivity dramatically from results in the literature as angular velocity changes provide further delineation of a fall event from other activities that may also experience high acceleration peaks.

\section{Introduction}

Fall events are the most significant cause of injury in the elderly. Falls result in many disabling fractures that could eventually lead to death due to complications such as infection or pneumonia. In the United States, more than onethird of elderly people over 65 years old fall at least once per year [1]. In 2008, hospital emergency rooms treated over 2.1 million nonfatal fall injuries among older adults of which $25 \%$ were hospitalized. Unfortunately, with age, chances of falling increases and injuries are exacerbated. Injuries due to falls for those over 85 years old were four times greater than in adults aged 65 to 74 . Further, once a person falls, they are likely to fall again.

While falls in the elderly have a direct health effect, another key effect is related to financial costs associated with the incident. In 2000, costs associated with falls in the elderly were estimated by the CDC to be approximately $\$ 20$ billion and to increase to $\$ 54.9$ billion by 2020 [2]. Half of elderly fallers are not able to get up by themselves [3]. On average, elderly fallers lie helplessly for more than 10 minutes with 3\% waiting for more than one hour before receiving assistance [4]. These long lies often result in hospitalizations, institutionalization, and high morbidity-mortality rates. Research by Bertera et al. [5] has shown that the elderly are interested in new technologies that support their health, independence, and safety, especially as it supports their desire to age-in-place [6].

Personal Emergency Response Systems (PERS) have been developed to facilitate calling for help after a fall event. However, in severe cases of an emergency, the individual may not be able to activate the PERS alarm. Recent reports show that $80 \%$ of elderly persons who were unable to get up after a fall did not use their PERS device to call for help $[7,8]$. For this reason, automatic fall detection is an active area of research. Many falls research strategies using accelerometers primarily 
utilize changes in acceleration magnitude to determine falls. When the acceleration exceeds a critical threshold, the fall is detected $[9,10]$. These systems successfully detect falls with sensitivities greater than $85 \%$ and specificities between 88 and $94 \%$. However, focusing only on large acceleration can result in many false positives as other activities such as sitting, running, and jumping may also result in large peak accelerations. For example, Huynh et al. [11] have shown that average peak acceleration during running (2.3-2.8 g) overlaps with the range observed during falls $(2.4-5.4 \mathrm{~g})$. This overlap reduces the specificity (i.e., detection of false positives) of purely accelerometer-based algorithms.

Other fall detection algorithms rely on detection of body orientation (i.e., prone) after a fall. However, these strategies may be affected by activities with similar postures (i.e., sleeping, reclining) and are less effective when a person's fall posture is not horizontal. Furthermore, additional studies have used complex algorithms such as support vector machine (SVM) [12] and Markov models [13] to detect falls. The robustness of these systems has not been demonstrated. They are also limited by excessive use of computational resources and cannot respond in real-time. While computational complexities can potentially be offloaded to cloud-based services, real-time and continuous access to the cloud may be unreliable especially in rural communities.

Unlike other prior works, this project proposes using a combination of accelerometer and gyroscope sensors for robust fall detection. While the accelerometer provides valuable information regarding body inertial changes due to impact, the gyroscope provides unique information regarding the body's rotational velocity during a fall event. A fall event produces both high acceleration and angular velocity, the combination of which is not observed during normal daily activities. The thrust of this work is to develop and optimize an algorithm for robust fall detection while simultaneously optimizing sensitivity and specificity.

\section{Methodology}

In prior studies [11], our team has developed a wireless sensor system and an algorithm to identify fall events from other daily activities such as walking, running, sitting, and climbing stairs. The system includes a wireless sensor system (WSS) comprised of a 3-axis accelerometer and gyroscope. Figure 1 shows the overall schematic of system. The WSS transmits and receives real-time accelerometer and gyro data during the fall. The detection algorithm is based on a simple threshold detection method using accelerometer thresholds found in the literature. Gyroscope threshold was determined experimentally using preliminary data collected.

2.1. Wireless Sensor System (WSS). The wireless sensor system (Figure 2) contains a sensor module (Figure 2(a)), Micro Control Unit (MCU), and Wi-Fi module to sense body orientation and activity data, control data flow, and transmit/ receive data, respectively. The WSS is placed at the center of chest (Figure 2(c)).

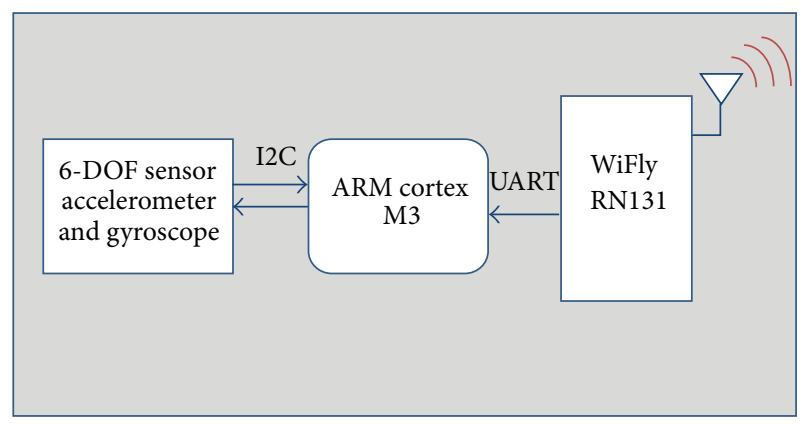

FIGURE 1: The schematic of wireless sensors system.

2.1.1. Sensor Module. Since our system uses both acceleration and angular velocity to detect falls, we choose to use the 6-DOF module with small size and power requirements. It includes a triaxial accelerometer ADXL345 and a triaxial gyroscope ITG3200 (Figure 2(a)). Acceleration measurement range of ADXL345, with 13-bit resolution and $4 \mathrm{mg} / \mathrm{LSB}$, is up to $\pm 16 \mathrm{~g}$. Additionally, the ITG-3200 gyroscope can capture the angular velocity between $\pm 2000^{\circ} / \mathrm{sec}$. Both sensors are connected with the MCU via I2C digital interface port.

2.1.2. Micro Control Unit (MCU) Module. The MCU for the control system is an LPC 1786 (NXP semiconductors), an ARM Cortex-M3 32-bit based microcontroller specifically designed for embedded applications requiring a high level of integration and low power dissipation. The chip can operate up to $100 \mathrm{MHz} \mathrm{CPU}$ frequency. In addition, the UART interface provides the sampling frequency up to $4 \mathrm{Mb} / \mathrm{s}$.

2.1.3. Wireless Module. The Wifly RN131 module is a standalone Wi-Fi module operating at a fully integrated $2.4 \mathrm{GHz}$ and providing an IP stack with IEEE $802.11 \mathrm{~b} / \mathrm{g}$ standard. The RN131 can operate with communication speed up to $11 \mathrm{Mbps}$. Due to its small form factor and extremely low power consumption, it is perfect for mobile wireless applications with portable battery operated devices. Additionally, its UART hardware interfaces for connecting with MCU can operate up to $1 \mathrm{Mbps}$ data rate.

2.1.4. Collection Program. The data collection program is written in Matlab (Mathworks, Inc., Natick, MA) to receive, display, and analyze real-time acceleration and angular velocity data from the WSS [11]. The program also stores data for later analyses.

2.2. Fall Detection Algorithm. The parameters used in analyses are similar to those proposed in previous studies $[10,14]$. The total sum acceleration vector, Acc, containing both static and dynamic acceleration components, is calculated from sampled data using

$$
\mathbf{A c c}=\sqrt{\left(A_{x}\right)^{2}+\left(A_{y}\right)^{2}+\left(A_{z}\right)^{2}},
$$




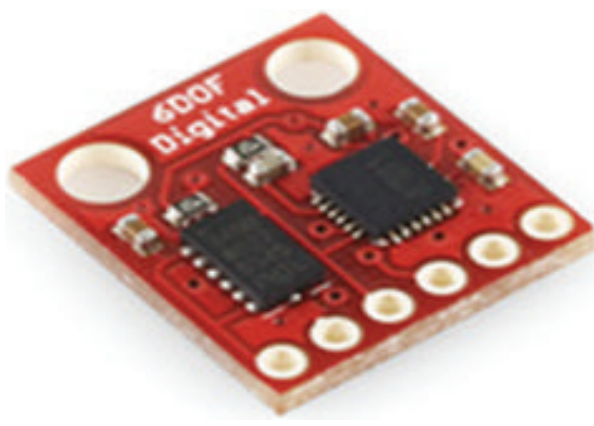

(a)

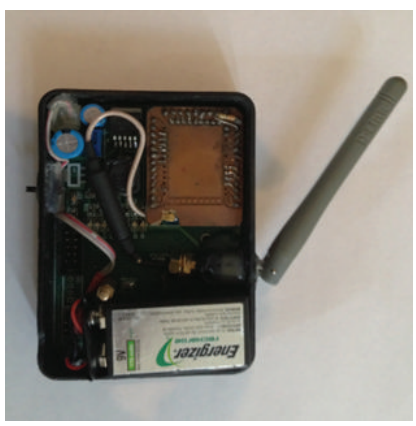

(b)

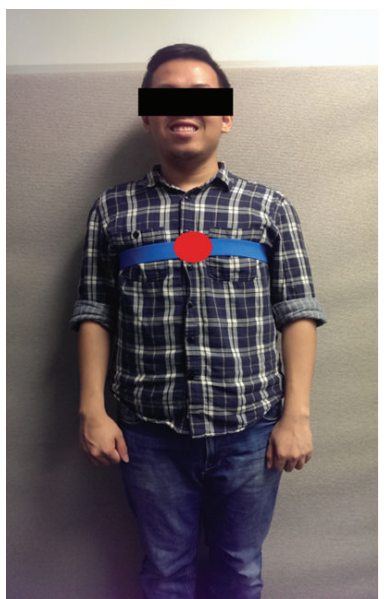

(c)

FIGURE 2: Fall detection system: (a) sensor module (6 DOF), (b) wireless sensor system (WSS), (c) system attached on center chest.

where $A_{x}, A_{y}$, and $A_{z}$ are the accelerations (g) in the $x, y$, and $z$ directions. Similarly, angular velocity is calculated from sampled data as indicated in the following:

$$
\boldsymbol{\omega}=\sqrt{\left(\omega_{x}\right)^{2}+\left(\omega_{y}\right)^{2}+\left(\omega_{z}\right)^{2}}
$$

where $\omega_{x}, \omega_{y}$, and $\omega_{z}$ are angular velocities in $x, y$, and $z$ directions.

When stationary, the acceleration magnitude, Acc, from triaxial accelerometer is constant $(+1 \mathrm{~g})$, and angular velocity is $0^{\circ} / \mathrm{s}$. When the subject falls, the acceleration is rapidly changing and the angular velocity produces a variety of signals along fall direction. Critical thresholds in the acceleration and angular velocity are then used for determining a fall event. These critical thresholds are defined and derived as follows [15].

(i) Lower fall threshold (LFT): local minima for the Acc resultant of each recorded activity are referred to as the signal lower peak values (LPVs). The $\mathrm{LFT}_{\text {acc }}$ for the acceleration signals is set at the level of the smallest magnitude lower fall peak (LFP) recorded.

(ii) Upper fall threshold (UFT): local maxima for the Acc resultant of each recorded activity are referred to as the signal upper peak values (UPVs). The UFT for each of the acceleration $\left(\mathrm{UFT}_{\mathrm{acc}}\right)$ and the angular velocity $\left(\mathrm{UFT}_{\text {gyro }}\right)$ signals are set at the level of the lowest upper fall peak (UFP) recorded for the Acc and $\omega$, respectively. The $\mathrm{UFT}_{\text {acc }}$ is related to the peak impact force experienced by the body segment during the impact phase of the fall.

Fall detection algorithms using accelerometers are normally divided into two groups, one based on $\mathrm{LFT}_{\text {acc }}$ comparison and the other based on $\mathrm{UFT}_{\text {acc }}$ comparison. Although past research has achieved some significant results, accuracy of these strategies is still below desired levels. Bourke et al. [15] used $\mathrm{LFT}_{\text {acc }}$ and $\mathrm{UFT}_{\mathrm{acc}}$ and found the sensitivity and specificity of performance to be $83.33 \%$ and $67.08 \%$, respectively.

In this study, we use both $\mathrm{LFT}_{\text {acc }}$ and $\mathrm{UFT}_{\text {acc }}$ in combination with the $\mathrm{UFT}_{\text {gyro }}$ for fall detection. We determine the LFT and UFT for both the acceleration and angular velocity based on collected experimental data. Our proposed algorithm is shown in Figure 3. First, the instantaneous acceleration magnitude, Acc, is computed and compared to the $\mathrm{LFT}_{\text {acc }}$. When Acc falls below the LFT acc threshold, data from the next $0.5 \mathrm{~s}$, period referred to as fall window, is compared to the UFT for both the acceleration and angular velocity vector. The fall window period was obtained from the literature $[16,17]$. Within the fall window, if both magnitudes of acceleration and angular velocity are above the $\mathrm{UFT}_{\mathrm{acc}}$ and 


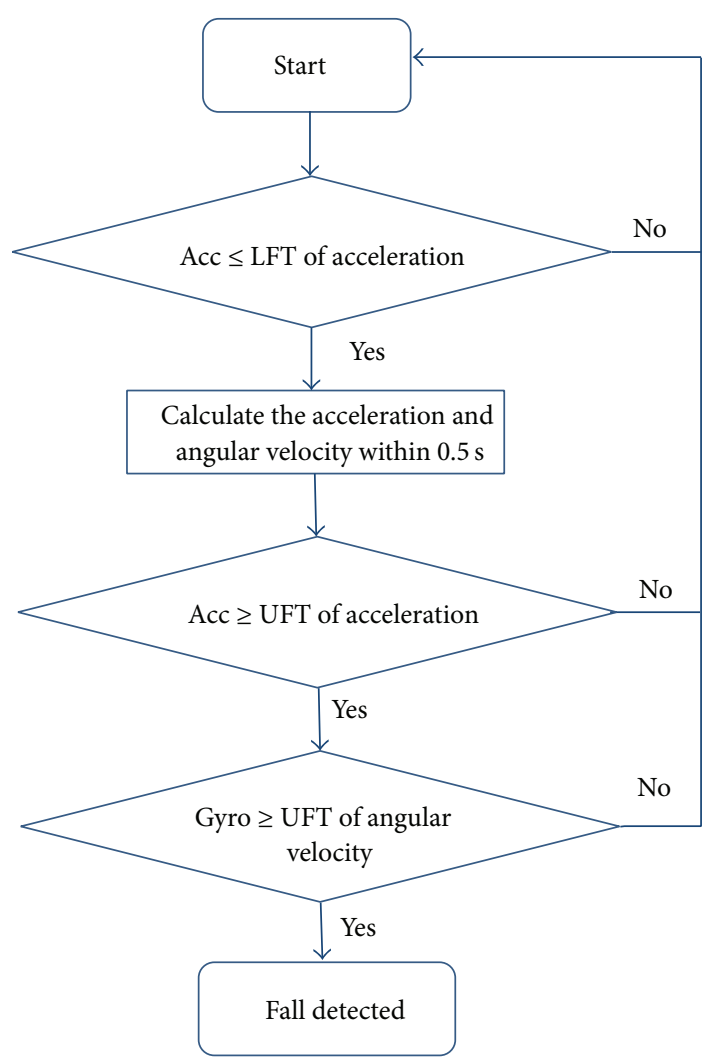

FIGURE 3: Combined accelerometer and gyroscope detection schema.

$\mathrm{UFT}_{\text {gyro }}$, respectively, then a fall is detected. If only one or neither is observed, a fall is not indicated.

Robustness of the proposed algorithm is evaluated by measuring sensitivity and specificity typically defined as

$$
\begin{aligned}
& \text { Sensitivity }=\frac{\text { No.TP }}{\text { No.TP }+ \text { No.FN }}, \\
& \text { Specificity }=\frac{\text { No.TN }}{\text { No.TN }+ \text { No.FP }},
\end{aligned}
$$

where the following happen.

(i) \# True positive (No.TP): fall occurs; device detects it.

(ii) \# False positive (No.FP): device detects fall; fall did not occur.

(iii) \# True negative (No.TN): normal movement (i.e., no fall); device does not declare a fall.

(iv) \# False negative (No.FN): fall occurs; device does not detect it.

Difficulty lies in determining the optimal thresholds for $\mathrm{LFT}_{\mathrm{acc}}, \mathrm{UFT}_{\mathrm{acc}}$, and $\mathrm{UFT}_{\text {gyro }}$. Improperly low threshold selection may lead to increased occurrences of false positives detected during daily activities, resulting in lower specificity. Alternatively, too stringent threshold selection may cause failure of fall detection altogether leading to increased false negatives and decreased system sensitivity.

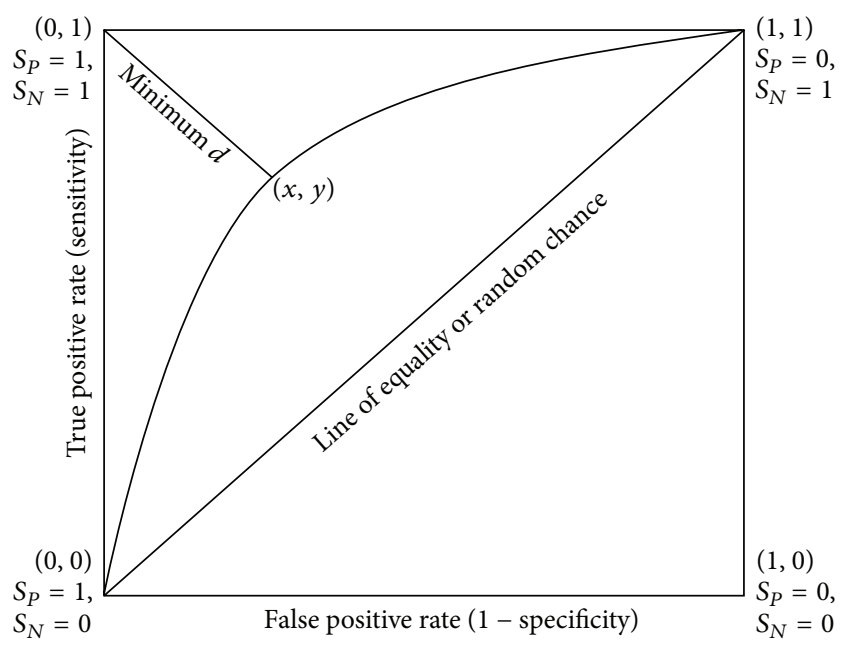

Figure 4: The ROC curve. Optimal threshold shown as minimum distance, $d$, to point $(0,1)$ where sensitivity $\left(S_{N}\right)$ and specificity $\left(S_{P}\right)$ are $100 \%$ and $100 \%$, respectively. False positive rate equals $1-$ specificity as an algorithm that results in $100 \%$ false positive has $0 \%$ specificity and one that has $0 \%$ false positive rate results in $100 \%$ specificity.

Many algorithms determine the thresholds experimentally [18]. Likewise, this study determines these thresholds for optimal sensitivity and specificity. To optimize thresholds, sensitivity and specificity are calculated for all possible thresholds values from experimental data. The sensitivity is inversely related with specificity in the sense that sensitivity increases as specificity decreases across various thresholds. The receiver operating characteristic (ROC; Figure 4) curve displays the tradeoff between the sensitivity ( $y$-axis) and specificity ( $x$-axis). The optimal threshold is the point that can provide the minimum distance between the point $(0,1)$ and itself [19]. The distance is defined in (4), where $S_{N}, S_{P}$ are the sensitivity and specificity, respectively, as follows:

$$
d_{T}=\sqrt{\left(1-S_{N}\right)^{2}+S_{P}^{2}} .
$$

2.3. Experimental and Procedure. To collect falls data for development and optimization of the falls algorithm, experiments were performed on 27 young healthy subjects (male and female, age from 18 to 28 years, weight from 50 to $90 \mathrm{~kg}$, and height from 154.5 to $180.0 \mathrm{~cm}$ ) and 9 middle-age subjects (age from 38 to 56 years, weight from 55 to $87 \mathrm{~kg}$, and height from 160.0 to $185.0 \mathrm{~cm}$ ) at The Catholic University of America (Washington, DC). The research protocol was approved by the Human Subjects/Institutional Review Board (IRB) Committee. The WSS was attached to the center of the chest of each subject, determined as optimal location by our previous study [20]. Each subject was asked to perform movements such as standing, walking, sitting down/standing up, step, and run and 4 different falls tests, forward, backward, and right/left sideway fall. Falls were conducted onto a 36 -inch high jump cushion to prevent injury. 

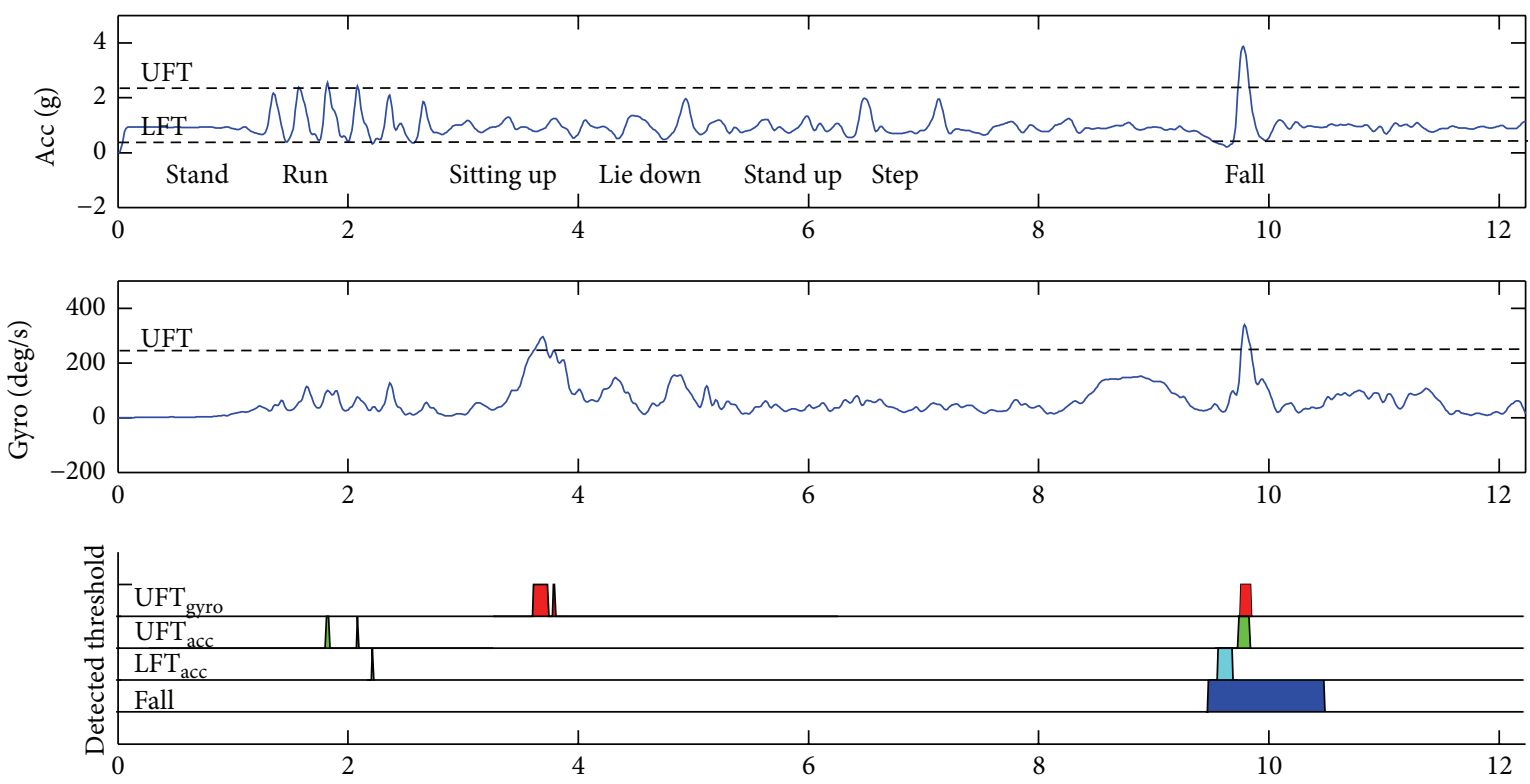

FIGURE 5: Display of Acc and $\omega$ for various activities: standing, running, sitting up, lying down, standing up, walking up steps, and falling. Also

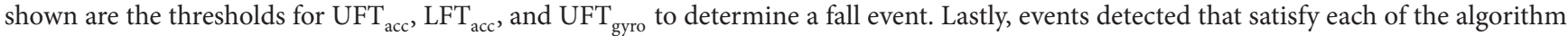
components are shown at the bottom. A fall event is denoted when all 3 thresholds are detected within the fall window.

\section{Results}

Figure 5 shows a typical example of the acceleration and angular velocity signals while performing different daily activities. Corresponding instances when the acceleration and angular velocity values exceed $\mathrm{LFT}_{\mathrm{acc}}, \mathrm{UFT}_{\mathrm{acc}}$, and UFT $_{\text {gyro }}$ during the various daily activities such as standing, running, sitting up, lying down, walking, stepping, and falling are also shown below the raw signals. During a fall event $(\sim 10 \mathrm{sec})$, the acceleration signal decreases from $1 \mathrm{~g}$ to cross below $\mathrm{LFT}_{\mathrm{acc}}$ and, within $0.5 \mathrm{~s}$, acceleration and angular velocity increase to cross above $\mathrm{UFT}_{\mathrm{acc}}$ and $\mathrm{UFT}_{\text {gyro }}$, almost simultaneously. With rigorous activities such as running, the peak of accelerations can reach critical LFT $_{\text {acc }}$ and $\mathrm{UFT}_{\mathrm{acc}}$ thresholds indicative of a fall event. However, the confirmatory UFT $_{\text {gyro }}$ threshold is never reached. For events such as lying down or sitting up, the $\mathrm{UFT}_{\text {gyro }}$ threshold is triggered, but the acceleration conditions are not met. Thus, the combination accelerometer and gyroscope sensor system complement one another for a more robust fall detection algorithm.

From the experimental data collected, a subset of the data (18 subjects) is analyzed to identify $\mathrm{LFT}_{\mathrm{acc}}$ and $\mathrm{UFT}_{\mathrm{acc}}$ of acceleration and $\mathrm{UFT}_{\text {gyro }}$ of angular velocity. After that, the remaining data is used for validation to determine the sensitivity and specificity of the algorithm. The mean and standard deviation values of minimum and maximum of acceleration and maximum of angular velocity are statistically for $90 \%$ and $99 \%$ confidence levels. Table 1 shows the mean and standard error for $\mathrm{LFT}_{\mathrm{acc}}, \mathrm{UFT}_{\mathrm{acc}}$, and $\mathrm{UFT}_{\text {gyro }}$ as well as values for $90 \%$ and $99 \%$ upper and lower confidence levels.

To improve system accuracy, the upper confidence limit between $90 \%$ and $99 \%(0.27 \mathrm{~g}-0.33 \mathrm{~g})$ for $\mathrm{LFT}_{\mathrm{acc}}$ and the
TABLE 1: Summary of critical threshold values for $\mathrm{LFT}_{\mathrm{acc}}, \mathrm{UFT}_{\mathrm{acc}}$, and $\mathrm{UFT}_{\text {gyro }}$ including mean \pm standard error, threshold values at upper and lower $90 \%$ confidence limits and at $99 \%$ upper and lower confidence limit.

\begin{tabular}{lccc}
\hline & $\mathrm{LFT}_{\mathrm{acc}}(\mathrm{g})$ & $\mathrm{UFT}_{\mathrm{acc}}(\mathrm{g})$ & $\mathrm{UFT}_{\mathrm{gyro}}\left({ }^{\circ} / \mathrm{s}\right)$ \\
\hline Mean \pm standard error & $0.18 \pm 0.06$ & $3.27 \pm 0.38$ & $382.1 \pm 98.9$ \\
Lower 90\% limit & 0.10 & 2.77 & 254.52 \\
Upper 90\% limit & 0.27 & 3.76 & 509.61 \\
Lower 99\% limit & 0.03 & 2.37 & 151.69 \\
Upper 99\% limit & 0.33 & 4.16 & 612.43 \\
\hline
\end{tabular}

lower confidence limit between $90 \%$ and $99 \%(2.37 \mathrm{~g}-2.77 \mathrm{~g})$ of $\mathrm{UFT}_{\mathrm{acc}}$ and $\mathrm{UFT}_{\text {gyro }}\left(151.7-254.5^{\circ} / \mathrm{s}\right)$ are used for the fall detection algorithm. To optimize the thresholds used for the final algorithm, the proposed algorithm is run at different levels for the ranges specified of $\mathrm{LFT}_{\mathrm{acc}}, \mathrm{UFT}_{\mathrm{acc}}$, and $\mathrm{UFT}_{\text {gyro }}$ and sensitivity and specificity values are calculated for the combined algorithm. Figure 6 shows a spatial mapping of sensitivity and specificity of system in the range of $\mathrm{LFT}_{\mathrm{acc}}(0.2 \mathrm{~g}-0.4 \mathrm{~g})$ and $\mathrm{UFT}_{\mathrm{acc}}(2 \mathrm{~g}-3 \mathrm{~g})$ of acceleration. As expected, there is a tradeoff between sensitivity and specificity with sensitivity increasing while specificity decreases, and vice versa. The optimized algorithm simultaneously maximizes both measures.

Because this study's goal is to develop a fall detection system with targeted accuracy over $90 \%$ for both sensitivity and specificity, three thresholds were initially chosen of $\mathrm{LFT}_{\mathrm{acc}}$, $\mathrm{UFT}_{\text {acc }}$, and $\mathrm{UFT}_{\text {gyro }} 0.26 \mathrm{~g}, 2.77 \mathrm{~g}$, and $254.5^{\circ} / \mathrm{s}$, respectively, based upon individual analyses from experimental data. Each threshold is then varied for the ranges above and sensitivity and specificity are calculated. From the ROC curves, the 


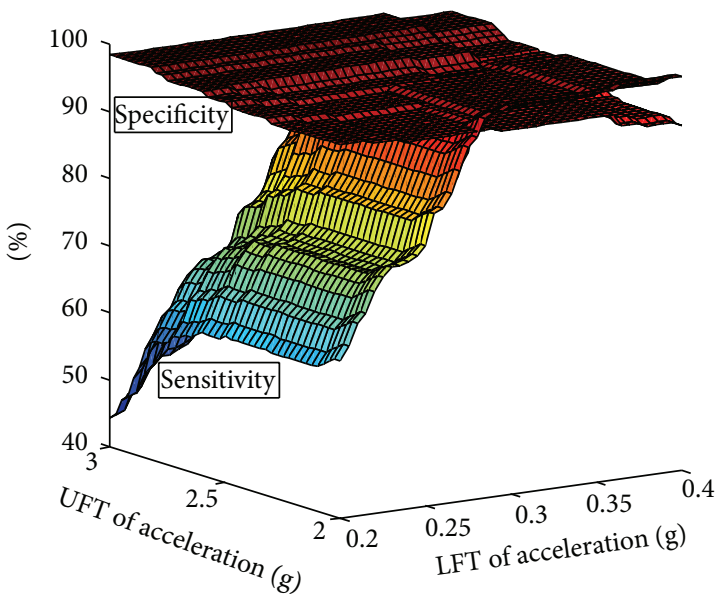

FIGURE 6: Graphical relationship between sensitivity and specificity with varying values of $\mathrm{LFT}_{\mathrm{acc}}$ and $\mathrm{UFT}_{\mathrm{acc}}$.

TABLE 2: Fall algorithm sensitivity and specificity without and with gyroscope while critical thresholds are set at $90 \%$ and $99 \%$ confidence levels.

\begin{tabular}{lcccc}
\hline & \multicolumn{2}{c}{$\begin{array}{l}\text { Accelerometer } \\
\text { without gyro }\end{array}$} & \multicolumn{2}{c}{ Accelerometer with gyro } \\
\hline $\begin{array}{l}\text { Confidence } \\
\text { level }\end{array}$ & $90 \%$ & $99 \%$ & $90 \%$ & $99 \%$ \\
\hline Sensitivity & $83.56 \%$ & $98.75 \%$ & $81.37 \%$ & $96.55 \%$ \\
Specificity & $96.12 \%$ & $75.93 \%$ & $99.38 \%$ & $89.50 \%$ \\
\hline
\end{tabular}

optimal threshold is chosen which minimizes distance $d$ in Figure 4.

Figure 7 shows the sensitivity versus threshold values for (a) $\mathrm{UFT}_{\text {gyro }}$, (b) $\mathrm{LFT}_{\text {acc }}$, and (c) $\mathrm{UFT}_{\text {acc }}$, respectively, and the corresponding ROC curves ((d)-(f)). The optimal threshold for correctly identifying a fall or nonfall is determined by minimizing distance $d$ in the ROC curves. For angular velocity (Figures 7(a) and 7(d)), this minimized distance $d$ is 0.063 and occurs when $\mathrm{UFT}_{\text {gyro }}$ is $240^{\circ} / \mathrm{s}$, corresponding to sensitivity of $95.5 \%$ and specificity of $97.1 \%(100 \%$ - specificity $=$ $2.9 \%)$. Similarly, Figures 7(b) and 7(e) represent the change of the sensitivity and specificity and ROC curves when LFT acc is varied. The minimized distance $d_{T}$ is 0.062 corresponding to an LFT range between $0.30 \mathrm{~g}$ and $0.35 \mathrm{~g}$ and results in $96 \%$ sensitivity and 95\% specificity. Lastly, Figures 7 (c) and 7(f) show the optimal UFT acc threshold $\left(d_{T}=0.059\right)$ of $2.4 \mathrm{~g}$ with corresponding sensitivity and specificity values of $94.9 \%$ and $97.5 \%$, respectively.

Table 2 provides a summary of results for fall sensitivity and specificity without (i.e., accelerometer only) and with inclusion of gyroscope information. In this table, critical thresholds for LFT and UFT are set at two confidence levels, $90 \%$ and $99 \%$. Since thresholds are set to desired confidence levels, no optimization strategy is implemented. Results show increasing confidence level from $90 \%$ to $99 \%$ increases sensitivity from $83.56 \%$ to $98.75 \%$, as expected, while dramatically decreasing specificity (from $96.12 \%$ to
TABLE 3: Fall algorithm sensitivity and specificity without and with gyroscope with ROC optimization strategy implemented.

\begin{tabular}{lcc}
\hline & Accelerometer without gyro & Accelerometer with gyro \\
\hline Sensitivity & $97.36 \%$ & $96.3 \%$ \\
Specificity & $82.72 \%$ & $96.2 \%$ \\
\hline
\end{tabular}

75.93\%) of accelerometer only algorithms. These results are similar to findings by others $[9,10]$. Addition of gyroscope information improves specificity by the ability to differentiate falls from other rigorous efforts. This is achieved with slightly diminished sensitivity compared to accelerometer only methods.

When critical thresholds are established and optimized using ROC analyses, one is able to achieve improved specificity while simultaneously achieving high levels of sensitivity for algorithms utilizing accelerometer only and with a combined accelerometer and gyroscope strategy (Table 3). For the combined accelerometer and gyroscope sensor plus optimization strategy, sensitivity and specificity for fall detection were $96.3 \%$ and $96.2 \%$, respectively.

\section{Discussion}

This study investigates a wireless sensor system that combines measures of acceleration and angular velocity of body to detect and differentiate falls from daily activities. In this study, 36 subjects performed four different types of falls as well as daily activities for a total of 9 different activities. Half the experimental dataset was used to develop and train the algorithm and to determine critical thresholds, while the other half was used to validate and test the final fall detection algorithm.

Tolkiehn et al. [21] reported results of an accelerometer only algorithm of $83.3 \%$ sensitivity and $79.1 \%$ specificity to detect falls. Chen et al. [22] reported achieving 94\% specificity with their fall detection algorithm, but with low sensitivity (76\%). Bourke et al. [23] recently proposed an algorithm with high sensitivity (97\%), but with a cost of lower specificity (83\%). Others have shown similar tradeoff between achieving high specificity [88-94\%] and decreased detection of falls (sensitivity $=85 \%)[9,10,24]$.

In this study, following an optimization schema using ROC curves and iterative analyses of sensitivity and specificity, we were able to determine critical thresholds for $\mathrm{LFT}_{\mathrm{acc}}$, $\mathrm{UFT}_{\text {acc }}$, and $\mathrm{UFT}_{\text {gyro }}$ that simultaneously result in high sensitivity and specificity of fall detection. These optimized values were $0.30 \mathrm{~g}-0.35 \mathrm{~g}, 2.4 \mathrm{~g}$, and $240^{\circ} / \mathrm{s}$, respectively. Results demonstrate the effectiveness of the combined accelerometer with gyroscope algorithm that is optimized for sensitivity and specificity compared to traditional accelerometer only strategies of fall detection. The optimized, combined sensor schema achieves both high sensitivity (96.3\%) and high specificity (96.3\%), simultaneously, much higher than prior results reported in the literature. The increased sensitivity is achieved by inclusion of gyroscope information which provides valuable additional information on body rotation in order to differentiate a fall event from other high impact 


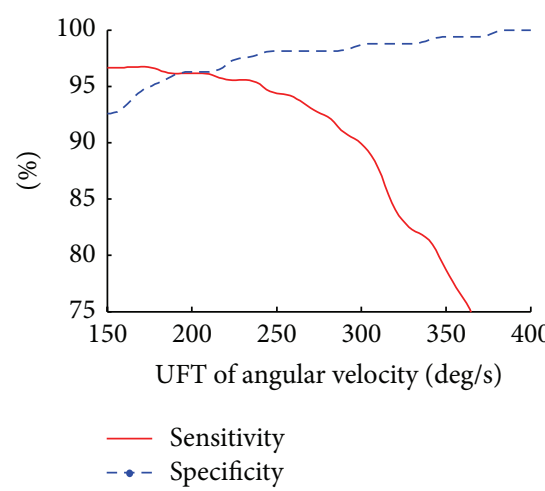

(a)

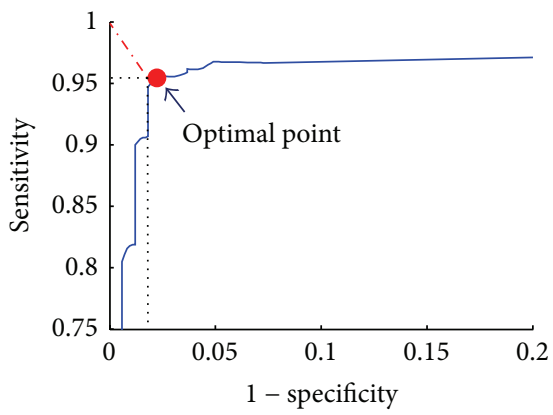

(d)

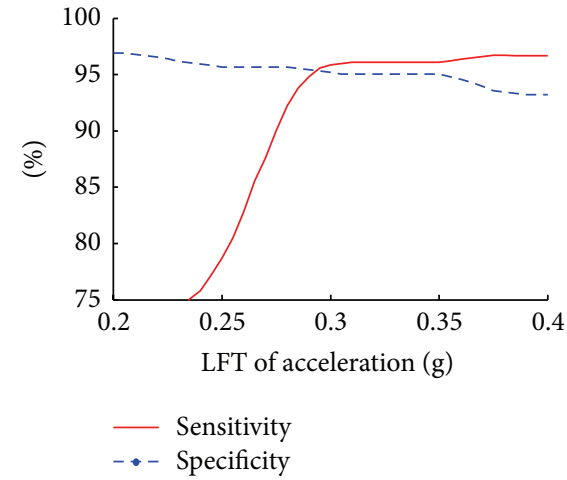

(b)

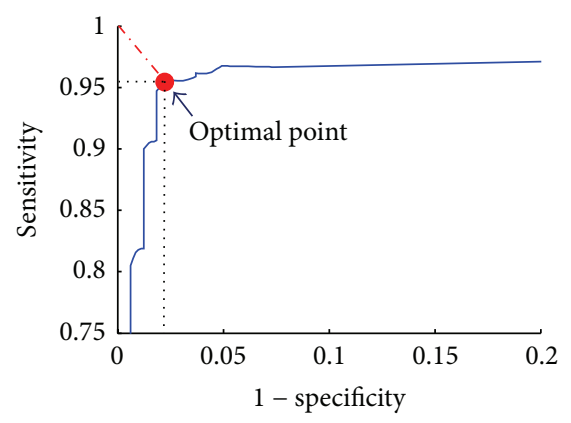

(e)

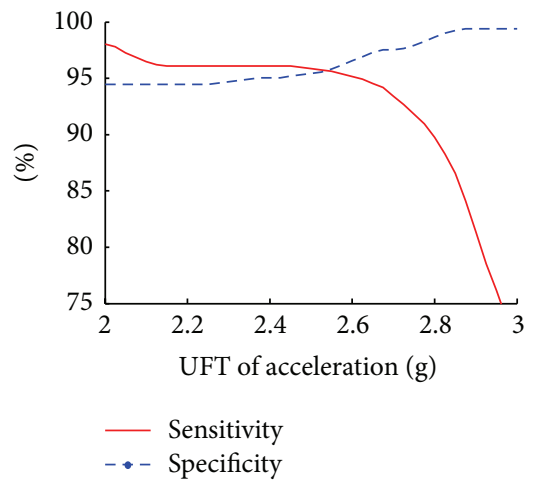

(c)

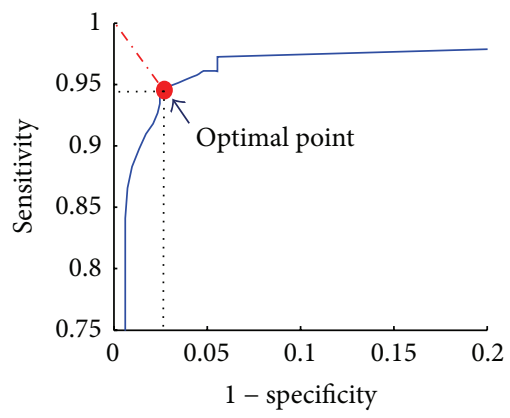

(f)

FIGURE 7: Representative curves of sensitivity versus threshold values (1st row) for (a) $\mathrm{UFT}_{\text {gyro }}$, (b) $\mathrm{LFT}_{\text {acc }}$, and (c) UFT acc and corresponding ROC curves (2nd row; (d)-(f)). Minimum distance point, $d$, is shown in each ROC curve with sensitivity ( 95\%).

activities such as running and jumping. The optimization strategy results in dramatic increases in specificity while only slightly sacrificing fall detection sensitivity.

The addition of gyroscope information reduces the occurrence of false positives resulting from rigorous activities such as running and jumping. While these activities produce high acceleration peaks similar to a fall, rotational angular velocities are much lower than seen in fall events and therefore are not triggered by the proposed algorithm. The inclusion of a check for body angular velocity threshold dramatically improves specificity from $82.72 \%$ without gyroscope to $96.2 \%$ with gyroscope. As there is a tradeoff between sensitivity and specificity, optimization of key thresholds via ROC analyses provides a systematic strategy for maximizing both sensitivity and specificity simultaneously.

\section{Conclusion and Future Work}

Fall detection is an important focus area in elderly care and greatly affects health, wellness, and disability. In this study, an optimized fall detection algorithm was developed using acceleration and angular velocity data collected from a wireless sensor system located in the center chest. Using an optimization approach, critical thresholds are determined through this work that results in robust fall detection. The optimized algorithm was able to detect falls with high sensitivity (96.3\%) and specificity (96.2\%) compared to prior strategies by other researchers who were able to achieve either high sensitivity or high specificity, but not both. The methodological approach followed in this effort also provides a systematic approach to selection of critical thresholds for fall detection that simultaneously optimizes both sensitivity and specificity. The proposed method is a simple threshold method and, therefore, is easy to port to wearable, electronic devices or inclusion in other mobile health platforms.

One limitation of this study is that the thresholds and algorithms were developed using data obtained from young healthy subjects and, for safety reasons, in a laboratory setting falling onto a cushioned mat. We expect that actual data from elderly individuals falling onto normal hard floors would experience higher peak accelerations and potentially higher angular velocities as these falls would be unexpected. Therefore, we expect the $\mathrm{LFT}_{\mathrm{acc}}$ UFT $_{\mathrm{acc}}$, and UFT $\mathrm{Uyro}_{\text {gyr }}$ threshold values from this work would still apply for real-world scenarios. While the system may be effective in detection of falls, it may be less effective in detection of near falls another clinically important area of falls research.

The system proposed herein is for a dedicated, wearable fall detection sensor system. Integration and deployment of the algorithm onto a smartphone, smartwatch device, or other mobile communication systems would be beneficial not only in detection of a fall, but also in alerting caregivers and other providers to the fall event. In these instances, the critical thresholds for the detection algorithm require further study. We and others have shown peak acceleration measures during a fall event vary considerably with position on the 
body $[16,20]$. Regardless, the proposed strategy of combining accelerometer and gyroscope measures is still expected to result in a robust fall detection algorithm.

\section{Conflict of Interests}

The authors declare that there is no conflict of interests regarding the publication of this paper.

\section{References}

[1] J. Y. Hwang, J. M. Kang, and H. C. Kim, "Development of novel algorithm and real-time monitoring ambulatory system using bluetooth module for fall detection in the elderly," in Proceedings of the 26th Annual International Conference of the IEEE Engineering in Medicine and Biology Society (IEMBS '04), vol. 1, pp. 2204-2207, San Francisco, Calif, USA, September 2004.

[2] National Center for Injury Prevention and Control and Centers for Disease Control and Prevention, Cost of Falls among Older Adults, National Center for Injury Prevention and Control, Centers for Disease Control and Prevention, 2013.

[3] A. Bueno-Cavanillas, F. Padilla-Ruiz, J. J. Jiménez-Moleón, C. A. Peinado-Alonso, and R. Gálvez-Vargas, "Risk factors in falls among the elderly according to extrinsic and intrinsic precipitating causes," European Journal of Epidemiology, vol. 16, no. 9, pp. 849-859, 2000.

[4] M. E. Tinetti, W. L. Liu, and E. B. Claus, "Predictors and prognosis of inability to get up after falls among elderly persons," The Journal of the American Medical Association, vol. 269, no. 1, pp. 65-70, 1993.

[5] E. M. Bertera, B. Q. Tran, E. M. Wuertz, and A. Bonner, "A study of the receptivity to telecare technology in a communitybased elderly minority population," Journal of Telemedicine and Telecare, vol. 13, no. 7, pp. 327-332, 2007.

[6] N. Farber, D. Shinkle, J. Lynott, W. Fox-Grage, and R. Harrell, Aging in Place: A State Survey of Livability-Policies and Practices, AARP Public Policy Institute, Washington, DC, USA, 2011.

[7] J. Fleming and C. Brayne, "Inability to get up after falling, subsequent time on floor, and summoning help: prospective cohort study in people over 90," British Medical Journal, vol. 337, no. 7681, pp. 1279-1282, 2008.

[8] D. Kunkel, R. M. Pickering, and A. M. Ashburn, "Comparison of retrospective interviews and prospective diaries to facilitate fall reports among people with stroke," Age and Ageing, vol. 40, no. 2, pp. 277-280, 2011.

[9] U. Lindemann, A. Hock, M. Stuber, W. Keck, and C. Becker, "Evaluation of a fall detector based on accelerometers: a pilot study," Medical and Biological Engineering and Computing, vol. 43, no. 5, pp. 548-551, 2005.

[10] M. Kangas, A. Konttila, P. Lindgren, I. Winblad, and T. Jämsä, "Comparison of low-complexity fall detection algorithms for body attached accelerometers," Gait \& Posture, vol. 28, no. 2, pp. 285-291, 2008.

[11] Q. T. Huynh, U. D. Nguyen, S. V. Tran, A. Nabili, and B. Q. Tran, "Fall detection using combination accelerometer and gyroscope," in Proceedings of the International Conference on Advances in Electronics Devices and Circuits (EDC '13), Kuala Lumpur, Malaysia, 2013.

[12] T. Zhang, J. Wang, L. Xu, and P. Liu, "Fall detection by wearable sensor and one-class SVM algorithm," in Intelligent Computing in Signal Processing and Pattern Recognition: International Conference on Intelligent Computing, ICIC 2006 Kunming, China, August 16-19, 2006, vol. 345 of Lecture Notes in Control and Information Sciences, pp. 858-863, Springer, Berlin, Germany, 2006.

[13] P. Jayachandran, T. F. Abdelzaher, J. A. Stankovic, and R. K. Ganti, "SATIRE: a software architecture for smart attire," in Proceedings of the 4th International Conference on Mobile Systems, Applications and Services (MobiSys '06), pp. 110-123, June 2006.

[14] J. Klenk, C. Becker, F. Lieken et al., "Comparison of acceleration signals of simulated and real-world backward falls," Medical Engineering \& Physics, vol. 33, no. 3, pp. 368-373, 2011.

[15] A. K. Bourke, K. J. O’Donovan, and G. ÓLaighin, “The identification of vertical velocity profiles using an inertial sensor to investigate pre-impact detection of falls," Medical Engineering \& Physics, vol. 30, no. 7, pp. 937-946, 2008.

[16] A. Ibrahim and M. Younis, "Simple fall criteria for MEMS sensors: data analysis and sensor concept," Sensors, vol. 14, no. 7, pp. 12149-12173, 2014.

[17] M. Popescu, B. Hotrabhavananda, M. Moore, and M. Skubic, "VAMPIR - an automatic fall detection system using a vertical PIR sensor array," in Proceedings of the 6th International Conference on Pervasive Computing Technologies for Healthcare (PervasiveHealth '12), pp. 163-166, San Diego, Calif, USA, May 2012.

[18] C. Dinh, D. Tantinger, and M. Truck, "Automatic emergecy detection using commercial accelerometers and knowledge," Computers in Cardiology, vol. 36, pp. 485-488, 2009.

[19] R. Kumar and A. Indrayan, "Receiver operating characteristic (ROC) curve for medical researchers," Indian Pediatrics, vol. 48, no. 4, pp. 277-287, 2011.

[20] Q. T. Huynh, U. D. Nguyen, S. V. Tran, and B. Q. Tran, "Optimum location for sensors in fall detection," in Proceedings of the International Conference on Green and Human Information Technology, 2013.

[21] M. Tolkiehn, L. Atallah, B. Lo, and G.-Z. Yang, "Direction sensitive fall detection using a triaxial accelerometer and a barometric pressure sensor," in Proceedings of the Annual International Conference of the IEEE Engineering in Medicine and Biology Society (EMBC '11), pp. 369-372, Boston, Mass, USA, August-September 2011.

[22] J. Chen, K. Kwong, D. Chang, J. Luk, and R. Bajcsy, "Wearable sensors for reliable fall detection," in Proceedings of the 27th Annual International Conference of the Engineering in Medicine and Biology Society (IEEE-EMBS '05), pp. 3551-3554, September 2005.

[23] A. K. Bourke, P. van de Ven, M. Gamble et al., "Evaluation of waist-mounted tri-axial accelerometer based fall-detection algorithms during scripted and continuous unscripted activities," Journal of Biomechanics, vol. 43, no. 15, pp. 3051-3057, 2010.

[24] I.-J. Kim, S. Im, E. Hong, S. C. Ahn, and H.-G. Kim, "ADL classification using triaxial accelerometers and RFID," in Proceedings of the International Conference on Ubiquitous Computing Convergence Technology, 2007. 

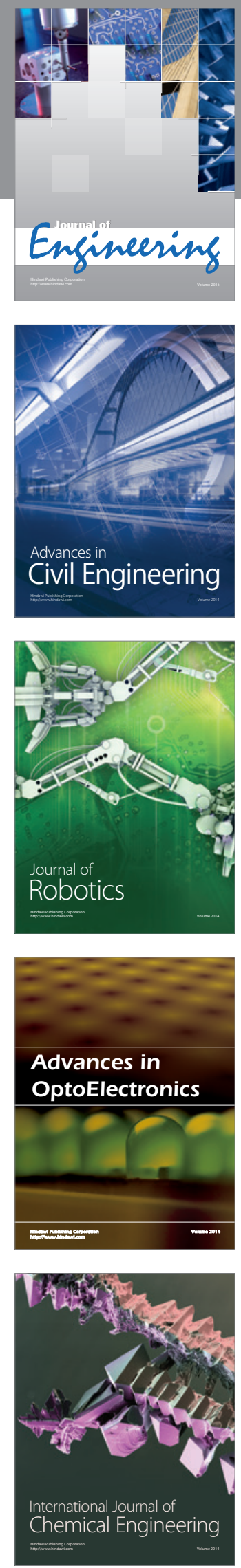

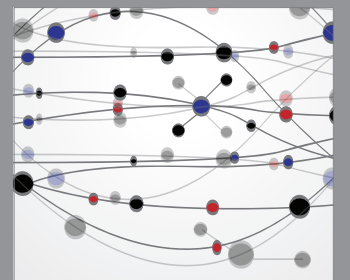

The Scientific World Journal
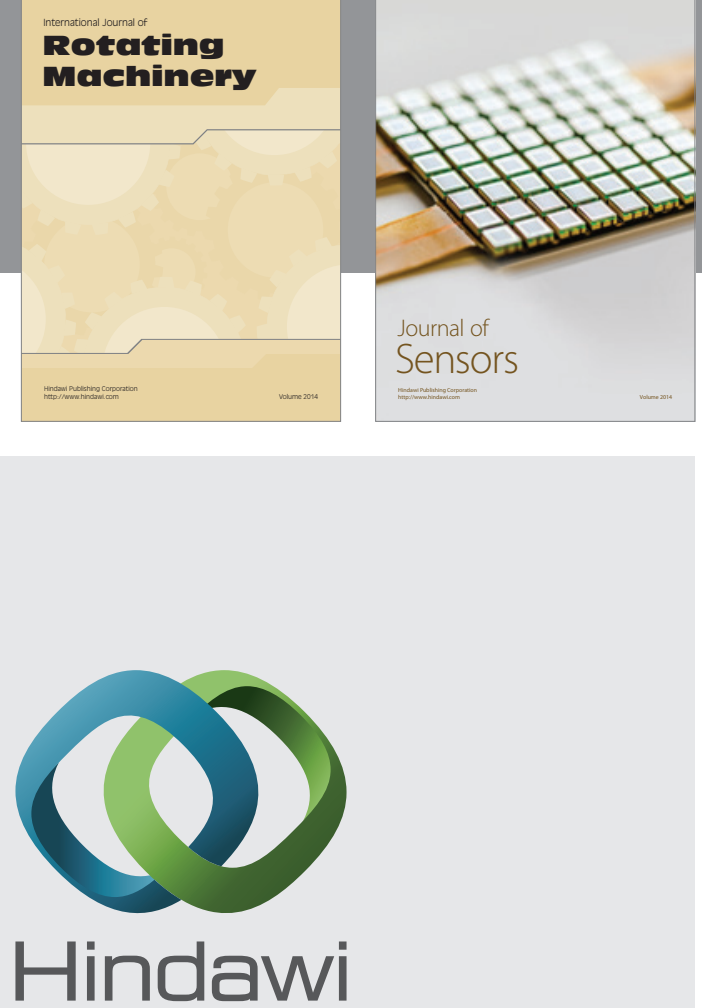

Submit your manuscripts at http://www.hindawi.com
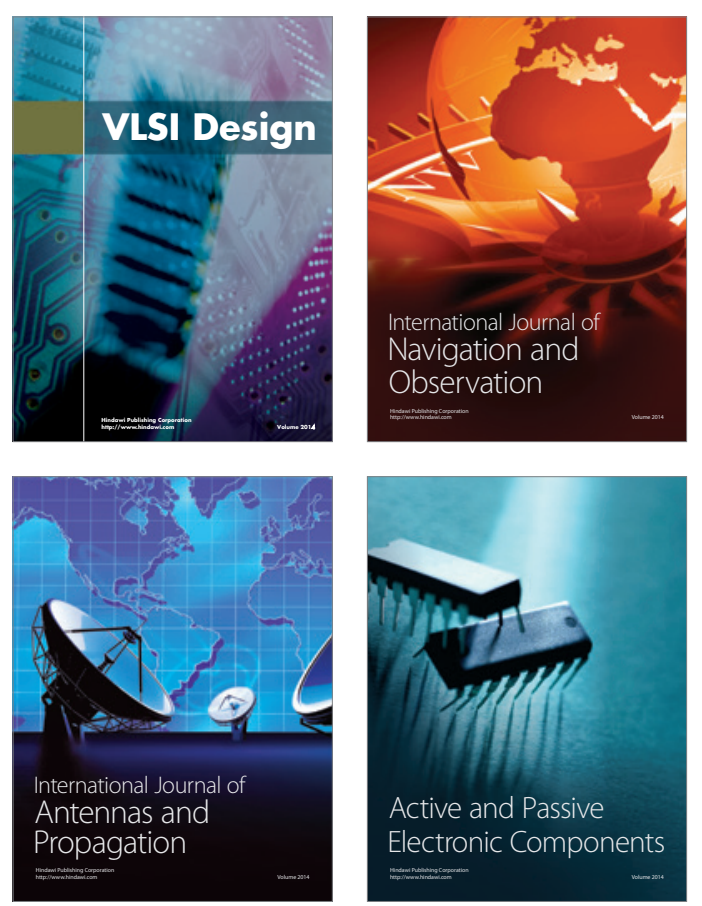
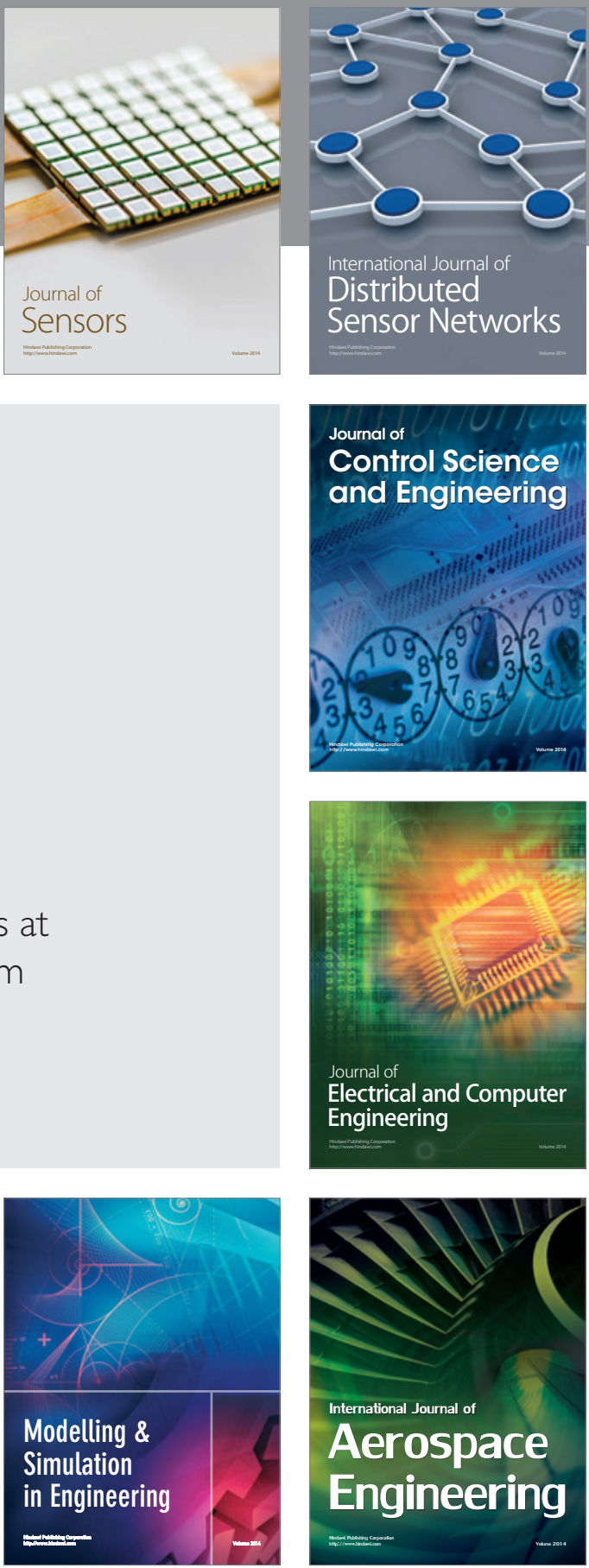

Journal of

Control Science

and Engineering
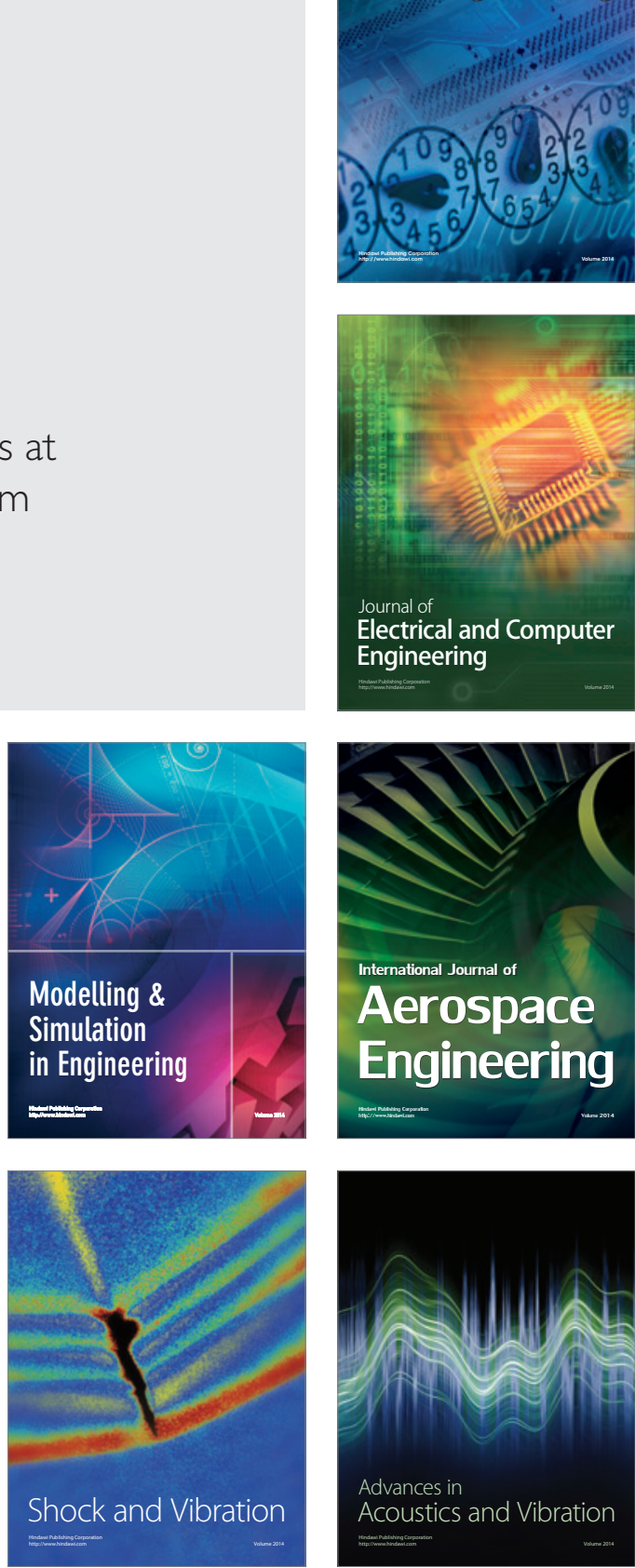